\title{
Does an Innovative Paper-Based Health Information System (PHISICC) Improve Data Quality and use in Primary Health Care? Protocol of a Multi-Country, Cluster Randomised Controlled trial in Sub-Saharan African Rural Settings.
}

Xavier Bosch-Capblanch ( $\nabla$ x.bosch@unibas.ch )

Institute. Basel, Switzerland. And University of Basel https://orcid.org/0000-0002-4469-0395

Angela Oyo-lta

Department of Community Medicine, University of Calabar

Artur Manuel Muloliwa

Faculty of Health Sciences, University of Lúrio

Richard B Yapi

Centre Suisse de Recherches Scientifiques en Cote d'Ivoire

Christian Auer

Swiss Tropical and Public Health Institute: Schweizerisches Tropen- und Public Health-Institut

Mamadou Samba

Ministère de la Santé et de l'Hygiène Publique: Ministere de la Sante et de l'Hygiene Publique

Suzanne Gajewski

Swiss Tropical and Public Health Institute: Schweizerisches Tropen- und Public Health-Institut

Amanda Ross

Swiss Tropical and Public Health Institute: Schweizerisches Tropen- und Public Health-Institut

L Kendall Krause

Bill \& Melinda Gates Foundation

Nnette Ekpenyong

Department of Community Medicine. University of Calabar Teaching Hospital

Ogonna Nwankwo

University of Calabar

Anthonia Ngozi Njepuome

Swiss Tropical and Public Health Institute: Schweizerisches Tropen- und Public Health-Institut

Sofia Mandjate Lee

Swiss Tropical and Public Health Institute: Schweizerisches Tropen- und Public Health-Institut

Jahit Sacarlal

Universidade Eduardo Mondlane Faculdade de Medicina

Tavares Madede

Department of Community Medicine. Universidade Eduardo Mondlane Faculdade de Medicina

Salimata Berté 
Centre Suisse de Recherches Scientifiques en Cote d'Ivoire

\section{Graça Matsinhe}

Ministério da Saúde: Ministerio da Saude

\section{Abdullahi Bulama Garba}

NPHCDA: National Primary Healthcare Development Agency

\section{David Brown}

BCGI LLC

\section{Study protocol}

Keywords: Decision-making, Health Information Systems, Primary Health Care, Sub-Saharan Africa, Data quality, Quality of care, Human Centred Design

Posted Date: February 26th, 2021

DOI: https://doi.org/10.21203/rs.3.rs-266052/v1

License: (c) (i) This work is licensed under a Creative Commons Attribution 4.0 International License. Read Full License 


\section{Abstract}

Background

Frontline health workers in remote, rural health facilities are the first contact of the formal health sector and are confronted with the need to make life-saving clinical and public health decisions. Appropriate health information systems (HIS) provide data to support the collection and use of data, thus facilitating decision-making. However, HIS focus on reporting and are unfit to support critical decisions at the peripheral level. Since data tools are paper-based in most primary health care settings, we have produced an innovative paper-based HIS (PHISICC), embracing all health care areas in primary health care, using a Human Centred Design, co-creation approach. The PHISICC tools aid decision-making, include recording and reporting and substitute the regular HIS tools. We are carrying out a cluster-randomised controlled trial in three African countries to assess the effects of PHISICC compared with the current systems, on data use and quality, quality of health care and health worker perceptions, in remote, rural settings.

\section{Methods}

We have selected study areas in rural zones of Côte d'Ivoire, Mozambique and Nigeria. Seventy health facilities in each country have been randomly allocated to using PHISICC tools or to continuing to use the regular HIS tools (35 per arm). We have selected three villages in the catchment area of each health facility to carry out surveys in 10 households each. Outcomes of interest include data quality and use, coverage of health services, health workers perceptions and other process and explanatory variables.

\section{Discussion}

We strive to contribute to producing robust evidence on health systems interventions, affecting people in remote, rural settings where the most vulnerable live. The PHISICC tools focus on decision-making rather than data and are meant to support health workers decisions as well as reporting to the higher levels of the system. Robust evidence on HIS can better find its way to high quality systematic reviews and guidance development to inform policy and practice.

Trial registration: Pan African Clinical Trials Registry - PACTR201904664660639. Registered 01/04/2019, https://pactr.samrc.ac.za/Search.aspx.

\section{Contribution To The Literature}

- Paper-based health information systems are widely used, and will continue to be used, in many Primary Health Care settings, particularly in remote, rural areas. Yet, little research is carried out on how to improve paper-based systems.

- We have developed new paper-based tools using a Human Centred Design approach, prioritising the decision-making experience of frontline health workers in the use of information support tools.

- Methodological and context challenges often preclude the implementation of robust experimental research studies in remote, rural areas of Sub-Saharan countries.

- Yet, robust evidence on HIS is more likely to gain the credibility of policy-makers and to make it into systematic reviews, guidance development and policy and practice. 


\section{Background}

Frontline health workers (HW) in remote, rural health facilities (HF) in many countries are the first contact with the formal health sector of the population and they are confronted with life-saving clinical and public health decisions on a daily basis. Decisions are made by exerting a balanced judgment on the information related to health care events, such as making the correct diagnoses or deciding on which vaccinations a child should receive on a given day. In order to properly handle this information, appropriate data support tools and processes are required, referred to as the health information system (HIS); or Routine HIS or Health Management Information System []. In reality, though, HIS are primarily designed to report aggregated health events to the higher tiers of the health systems rather than to inform decision-making at the point of care [].

Increasing pressure by donors and governments to collect more and more data has aggravated the situation, through the proliferation of data support tools that have overloaded frontline health workers compromising their capacity to deliver good quality of care and to delivery good quality data [, for higher level decision-making.

Promising 'quick fixes', such as the scale up of digital HIS, are taking a long time to implement and face enormous challenges related to infrastructure, equipment and services necessary to run them. Besides, research evidence on the effects of digital solutions remains patchy and inconsistent, even in high-income country settings, where complaints about computerisation of clinical care have been raised []. Hence, it is very likely that paper tools will remain a primary, if not unique, data support mechanism particularly in remote, rural HF in many countries.

PHISICC (Paper-based Health Information System in Comprehensive Care) is a multi-year, multi-country, mixedmethods research project that aims at producing and testing an innovative paper-based HIS to improve data quality and use, decision making and health outcomes, at Primary Health Care (PHC). It is being carried out in selected areas within Côte d'Ivoire, Mozambique and Nigeria. The project started in 2015, producing a systematic review on the effects of HIS interventions and a framework synthesis on how HIS are understood in the literature. These were followed by studies to characterise the existing HIS in the three countries. With these bodies of evidence, we engaged into a Human Centred Design (HCD) co-creative process with frontline HW to design an innovative HIS (PHISICC).

The impact of the PHISICC HIS on data quality and use, quality of health care and HW perceptions is being assessed concurrently in rural areas in the three countries. We describe the design of the trial here, consistent with CONSORT reporting guidelines [ and the extension for cluster randomised controlled trials (CRCT) [; see Additional file 1.

\section{Methods}

\section{Aim}

The aim of the trial is to address the research question: what are the effects of an innovative paper-based HIS (PHISICC) on data use and quality, quality of health and HW perceptions compared with the current HIS, in rural PHC settings?

\section{Study design}


The study is a CRCT in each of the three countries. In each setting, 70 health facilities are randomised to intervention or control (35 per arm). The intervention arm HF use the new PHISICC tools (substituting the usual HIS tools) and the control arm HF use the regular HIS tools. The trial is implemented in the real life contexts of HF carrying out their usual duties.

The CRCT are implemented in the real life contexts of HF carrying out their usual duties. The trials started between the end of 2019 and beginning of 2020, depending on the country, when the intervention was installed and the baseline surveys carried out; and will last till mid-2021.

\section{Study areas}

Ministries of Health $(\mathrm{MOH})$ officials in several countries were contacted before submitting the proposal to the funding agency in order to explore the willingness to engage in a project focusing on paper-based tools. Officials in several countries rejected the offer on the grounds of upcoming digitalisation plans of the HIS in the country. We partnered with $\mathrm{MOH}$ that found the research relevant to their context in three countries.

In each country, the eligibility criteria of study areas were that they had to belong to the operational area of research partners; contain a large enough number of health facilities and their catchment population; include vulnerable population (e.g. with low vaccination coverage, high childhood mortality); and be comparatively neglected in terms of infrastructure and services. We excluded areas with concurrent research or other types of activities that could conflict with the CRCT (such as the co-existence of another health-related study, massive developments in infrastructure or activities involving migration of the population, such as temporary work sites or changes in working sites) and areas with threats to safety or security that could jeopardise research activities.

The study areas are located in Adzopé, Agboville, Tiassalé and Sikensi districts (Côte d'Ivoire); in Funhalouro, Govuro, Homoine, Inhambane, Inharrime, Inhassoro, Mabote, Maxixe and Panda (Inhambane province, Mozambique); and in Yala Local Government Authority (Cross-River State, Nigeria).

\section{Eligibility of health facilities}

The intervention is implemented at the HF level. The eligibility criteria of the HF were that they had to be located in the study areas, belong to the governmental health sector and their main activity should be the delivery of PHC services. HF were excluded if they had specialised clinical services, inpatients, physicians providing care or with plans for staff turn-over involving intervention and control HF.

A 'master list' of eligible health facilities was prepared based on information provided by the $\mathrm{MOH}$ across all study areas. We aimed at selecting 70 of the eligible HF in each country, using simple random techniques in R I.

\section{Allocation and blinding}

Allocation of the $70 \mathrm{HF}$ per country into the intervention and control arms took place in a formal event, gathering research partners and $\mathrm{MOH}$ officials to offer transparency and promote study ownership by local and national authorities. Equally sized, folded pieces of paper with the names and codes of included HF written on them were introduced in an opaque receptacle where they were manually and blindly mixed. A second receptacle contained two equally sized pieces of paper, one with the word 'intervention' and another one with the word 'control'. A selected person in the meeting, not belonging to the research team, extracted one piece of paper at a time to reach half the number of included HF. Then, a paper was extracted from the second receptacle to assign those HF 
to the intervention or control arms. The rest of the papers were extracted as well to verify completeness and no duplication of names, and those HF assigned to the other arm.

Once HF were selected, all villages or settlements for each health facility catchment area were listed and three in each catchment area were selected. In practice, we selected all villages because the numbers were below (in Côte d'Ivoire) or just above (in Nigeria) the needs. For each village, we used Google satellite maps to identify and geolocate every visible roof. Where there were many houses per village (roughly, more than fifty or so), a researcher would mark four points in the map slightly beyond the northernmost, southernmost, easternmost and westernmost roofs seen and 30 random points were selected within that square. From the mapped points, 10 per village (with 10 more acting as reserve) were randomly selected and marked on another map used in the field for data collectors to approach households. Where technical problems impeded this approach in a given village, a field supervisor would rotate a bottle on the floor towards the centre of the village and would select at random 10 households in the direction pointed by the bottle, from the outer limit of the village till the centre [.

Blinding is only feasible for the research team members carrying out the CRCT data collection and the analyses of the CRCT findings. The intervention (i.e. paper tools) are by design very different from the existing system and it is not possible to blind participants or principal researchers.

We already had the agreement of the $\mathrm{MOH}$ and selected HF compliant with the inclusion criteria were provided with the intervention shortly after completing the baseline data collection. Therefore, recruitment as such took place at the same time of the allocation of HF into intervention and control arms.

\section{The intervention}

The PHISICC paper-based intervention is a full set of paper-based tools to support decision-making by frontline $\mathrm{HW}$. These are the only tools to be used by HW in the intervention arm. The PHISICC tools encompass the whole system (i.e. recording and reporting) and all clinical and public health care areas and are characterised by: a common visual language (e.g. spaces for digits and text), standardised formats across health care areas; support to critical data items (e.g. respiratory rate in infants); graphic artefacts to distinguish severity degrees of signs or symptoms; documentation of diagnoses and treatment decisions; and aides memoires, among others.

The PHISICC tools have been developed over 18 to 20 months prior to the CRCT, using a Human Centred Design approach []. A strength of the Human Centred Design approach is its ability to unlock the user's perspective so that designers can build solutions that are fully reality-based and work well. Co-creation groups were formed in each country with researchers, staff from partner institutions and healthcare workers, led by a team of professional designers. Based on co-creation, participatory processes, and Human Centred Design principles, many iterations took place between co-creation groups and end-users of the tools, the frontline HW, till reaching a design that considered and addressed the main issues raised by HW (i.e. usability, clarity, size of tools). The PHISICC tools have been produced in French for Côte d'Ivoire, in Portuguese for Mozambique and English for Nigeria, which are the official languages used in the health systems in the three countries; using the official logo of the MOHs. Health care areas covered include: family planning, antenatal care, including tetanus toxoid vaccination, delivery, post-natal care, vaccination, sick child, adults outpatient consultation, tuberculosis diagnosis and treatment, and HIV. Referral forms were also designed.

The PHISICC tools have three sub-components: registers, tallies and reports. Registers are formed by seven DINA3 and one DIN-A4 (for referrals) book covering all health care areas except for tuberculosis treatment, for which 
DIN-A3 cards where used. Register books have 100, 200 or 400 pages depending on the country and health care area. They are used to record individual clients' data for each health care event, either of clinical or public health nature. Some register books have clinical notes at the very beginning, as 'aide memoires', and an example of a filled-in form, to assist HW when doubting how to proceed.

Tallies are DIN-A3 single sheets which contain a list of the indicators to be transferred to higher levels of the health system, with a series of small ovals, grouped in fives, to mark with tally sticks with a pen. In contrast to the current systems that have no tallies or only for vaccination, tallies were created for all health care areas. In the middle-right side of the tally, a column accommodates cells aligned with the ovals to insert the count for each indicator; and in the far right of the sheet there is a replica of the count column, separated with a perforated line, which is detached and sent, as part of the monthly report to the higher level in the health system.

During three or four days, HW were trained on HIS before the start of the trial. In the intervention arm they were trained on the PHISICC tools; and the control arm received a refresher training about the regular tools, during the same number of days.

Additionally, given that the regular tools already contained information on past vaccination history of children still to complete their vaccination schedule, we created a mechanism to retrieve data of children's vaccination status to transcribe into the new vaccination register book in the intervention arm ('system transition').

Tools were endorsed by $\mathrm{MOH}$, printed in local printing companies and distributed to $\mathrm{HW}$ at the end of the training sessions. A digital spreadsheet was created to monitor consumption and order additional tools to cover health facility needs during the life of the trial.

\section{Outcomes}

There are five primary outcomes (Table 1). Vaccination adherence is defined as the total number of vaccine doses given during the trial period in the correct time interval to children over the total number of vaccine doses that should have been given during the same period. Antenatal care visits uptake will also be considered depending on the expected number of pregnancies in the study areas. Both are used as proxies for health outcomes in terms of protection against disease [] and prevention of pregnancy complications [. Data concordance is defined as the level of agreement of HIS indicators between (i) records, (ii) tallies and (iii) reports [3]. In terms of data use for decision making, we will estimate the diagnostics scope in the sick child (i.e. number of different diagnoses per child; and treatment appropriateness (i.e. number of prescribed treatments that are supported by a documented diagnosis). Health workers satisfaction will be assessed using a standardised questionnaire [,]. While the intervention targets HF, some of the outcomes are measured at the level of HF, and some from patients clustered within HF catchment areas. 
Table 1

Outcomes and parameters used to estimate the sample sizes.

\begin{tabular}{|c|c|c|c|c|c|c|}
\hline & Outcome name & Subjects & Definition & $\begin{array}{l}\text { Baseline } \\
\text { estimate }\end{array}$ & $\begin{array}{l}\text { Expected } \\
\text { change }\end{array}$ & Comments \\
\hline 1 & $\begin{array}{l}\text { Vaccination } \\
\text { adherence }\end{array}$ & $\begin{array}{l}\text { Children } \\
\text { under- } 1 \text { in the } \\
\text { households }\end{array}$ & $\begin{array}{l}\text { Number of } \\
\text { vaccines } \\
\text { given in the } \\
\text { previous } \\
\text { calendar year } \\
\text { over the } \\
\text { number of } \\
\text { vaccine due in } \\
\text { the same } \\
\text { period }\end{array}$ & $\begin{array}{l}75 \text { given per } \\
100 \text { due }\end{array}$ & $\begin{array}{l}\text { Increase of } 10 \\
\text { per } 100\end{array}$ & $\begin{array}{l}\text { Vaccines are } \\
\text { clustered } \\
\text { within } \\
\text { children, and } \\
\text { children } \\
\text { within HFs }\end{array}$ \\
\hline 2 & $\begin{array}{l}\text { Data } \\
\text { concordance }\end{array}$ & $\begin{array}{l}\text { Recording } \\
\text { tools in } \\
\text { health } \\
\text { facilities }\end{array}$ & $\begin{array}{l}\text { Number of } \\
\text { health care } \\
\text { events (e.g. } \\
\text { vaccinations, } \\
\text { antenatal care } \\
\text { consultations) } \\
\text { recounted in } \\
\text { the previous } \\
\text { calendar year } \\
\text { versus the } \\
\text { number of } \\
\text { health care } \\
\text { events } \\
\text { reported in the } \\
\text { same time } \\
\text { period }\end{array}$ & $\begin{array}{l}7 \text { recounted } \\
\text { for each } 10 \\
\text { reported [14] }\end{array}$ & $\begin{array}{l}\text { Increase of } 2 \\
\text { recounted }\end{array}$ & $\begin{array}{l}\text { A single } \\
\text { estimate can } \\
\text { be obtained } \\
\text { in each HF or } \\
\text { by time } \\
\text { periods (no } \\
\text { clustering) }\end{array}$ \\
\hline 3 & $\begin{array}{l}\text { Diagnostic } \\
\text { scope }\end{array}$ & $\begin{array}{l}\text { Records of } \\
\text { sick child } \\
\text { consultations }\end{array}$ & $\begin{array}{l}\text { Number of } \\
\text { diagnosis in } \\
\text { each sick } \\
\text { child } \\
\text { consultation } \\
\text { during the } \\
\text { previous } \\
\text { calendar year }\end{array}$ & $\begin{array}{l}1 \text { or } 2 \text { per } \\
\text { child }\end{array}$ & $\begin{array}{l}30-35 \% \text { with } \\
\text { more than } 1 \\
\text { diagnosis }\end{array}$ & $\begin{array}{l}\text { Individual } \\
\text { consultations } \\
\text { are clustered } \\
\text { within HF }\end{array}$ \\
\hline 4 & $\begin{array}{l}\text { Treatment } \\
\text { appropriateness }\end{array}$ & $\begin{array}{l}\text { Records of } \\
\text { sick child } \\
\text { consultations }\end{array}$ & $\begin{array}{l}\text { Number of } \\
\text { treatments } \\
\text { correctly } \\
\text { prescribed in } \\
\text { each sick } \\
\text { child } \\
\text { consultation } \\
\text { during the } \\
\text { previous } \\
\text { calendar year }\end{array}$ & $\begin{array}{l}\text { Half } \\
\text { appropriate } \\
\text { over all } \\
\text { consultations }\end{array}$ & $\begin{array}{l}\text { Increase to } \\
\text { three quarters } \\
\text { appropriateness }\end{array}$ & $\begin{array}{l}\text { Individual } \\
\text { consultations } \\
\text { are clustered } \\
\text { within HF }\end{array}$ \\
\hline 5 & $\begin{array}{l}\text { Health } \\
\text { workforce } \\
\text { satisfaction }\end{array}$ & $\begin{array}{l}\text { Health } \\
\text { workers }\end{array}$ & $\begin{array}{l}\text { Degree (score) } \\
\text { of } \\
\text { satisfaction } \\
\text { across all } \\
\text { health } \\
\text { facilities in } \\
\text { each arm, } \\
\text { with the } \\
\text { intervention }\end{array}$ & 5 out of 10 & 9 out of 10 & $\begin{array}{l}\text { Maybe two or } \\
\text { three health } \\
\text { workers can } \\
\text { be } \\
\text { approached } \\
\text { in each } \\
\text { health facility }\end{array}$ \\
\hline
\end{tabular}


Secondary outcomes are classified under the following domains: data quality, data user, mortality, HW experience, clients experience and resource consumption

- Data quality

- Completeness of recording and reporting in specific forms; i.e. prevalence of unduly missing data items; partograph used;

- accuracy of recorded figures in comparison to real events (e.g. physical counting of commodities, such as number of $500 \mathrm{mg}$ Paracetamol tablets as recorded versus number of $500 \mathrm{mg}$ Paracetamol tablets as counted;

- timeliness of reporting, as documented by time stamps in forms;

loss of data or data which does not reach the next upper administrative level.

- Data use

- in terms of knowledge (e.g. vaccines due based on date of birth; weight for length assessments);

- cases of different conditions properly treated in (e.g. diarrhoea cases given oral rehydration therapy according to national guidelines; pneumonia cases given appropriate antibiotic according to national guidelines;

- public health decisions: availability of lost to follow up lists or plans for vaccination, tuberculosis and or HIV/AIDS treatment control;

occurrence of stock outs of essential drugs

- Overall under-5s mortality and under-5s mortality excluding peri-natal mortality [].

- Health workers 'human experience' and satisfaction

- District health information officers' 'human experience'

- Clients 'human experience' and satisfaction

- Resources consumption (e.g. time use, costs)

intervention costs: tools, training, start-up;

time used for recording and reporting (e.g. time-motion study) I;

cost-effectiveness per unit of additional improvement in outcomes of interest.

In addition, we will consider 'explanatory outcomes' that will help to understand how the measured effects have taken place and why. We will look at the details of the interplay between the intervention, the system, the users and the context. Process indicators will be based on the documented activities that have taken place, from the conception of the intervention, up to its implementation, monitoring and evaluation. Process indicators may include: intervention set up and implementation, monitoring of the use of the intervention, special activities targeted at vulnerable populations, district reactions related to the intervention, handling of data coming from the new system, sustainability based on costs information and perceptions, alignment with national health policies and donor priorities. We will also explore health care services characteristics looking at generic indicators from health facilities, such human resources profiles and relations with the communities, population characteristics and system and context characteristics captured in early stages of the project, where data are available.

\section{Sample size calculations}


The required sample sizes for each primary outcome were determined using simulation. This allowed us to account for levels of clustering (Table 1). We used the regression models detailed in the data section to analyse the simulated trials and estimate the power. The simulation code was written in $\mathrm{R}$.

For each country, we required the probability of a type I error (rejecting the null hypothesis when it is actually true) a to be less than 0.05 and a power of $80 \%$.

The sampling frames are the study areas in each country, which include the health facilities and households in their catchment areas.

For vaccination adherence, using a sample size of $35 \mathrm{HF}$ per arm, we would have $80 \%$ power in each country to detect as significant a difference between a proportion of due vaccines given from $75 \%$ in the control to $85 \%$ in the intervention arms, assuming one child per household, 30 households per HF and a between-HF variation equivalent to a $k$ of 0.1 , where $k$ is equal to the standard deviation divided by the mean. The value of $k$ is unknown, but was chosen in line with general observations by Hayes and Bennet [].

For data quality outcomes, with $35 \mathrm{HF}$ per arm we would be able to detect as significant a difference from a ratio of 0.7 (reported : recorded) vaccinations in the control arm to 0.8 with the intervention with $80 \%$ power, assuming 100 recorded vaccinations per HF and a standard deviation of 0.1 in the ratios between HF.

In terms of diagnostic scope, we would be able to detect an increase in the proportion of child-visits with more than one diagnosis from $30-35 \%$ with $80 \%$ power with $35 \mathrm{HF}$ per arm, 60 records per HF and assuming a $\mathrm{k}$ of 0.1 [18].

We would be able to detect as significant an increase from $50 \%$ of treatments having a corresponding appropriate diagnosis to $60 \%$ with $80 \%$ power assuming $35 \mathrm{HF}$ per arm, 1 treatment per child, 25 children per HF and variation between HF corresponding to $k=0.1$ [22].

For the outcome related to health workers' satisfaction, we would be able to detect as significant an increase from $50 \%$ of health workers satisfied to $90 \%$, with $80 \%$ power assuming $35 \mathrm{HF}$, three health workers per $\mathrm{HF}$ and a variation between HF equivalent to $\mathrm{k}=0.1$.

In summary, in each country we require $35 \mathrm{HF}$ per arm, three HW per HF, 100 vaccination records per HF, 60 sick child records per health facility and 30 children per health facility catchment area.

\section{Data collection and management}

Data collection took place at baseline and will take place again at the end of the study. Data is collected from health facilities, from the households in the catchment areas of the included health facilities and also from district offices.

For data quality and data use outcomes, HF registers, tallies and reports will be scrutinised. For population based outcomes, we carry out household surveys at baseline and at end-line. We use standard approaches for these types of surveys [. Households are visited, the research project is briefly introduced and consent requested.. Ideally, mothers of alive children or women in child-bearing age were interviewed in order to obtain information on living children (i.e. vaccination history) and death events, respectively, using home-based records if available and accessible. Patients' satisfaction will be assessed using the PSQ-18 satisfaction questionnaire [,]. Essentially, the 
tool enables practitioners to investigate the extent to which their health care service meets the perceived needs of their client group and pinpoint areas for improvement [19]. The interview will be conducted with consenting patients as close to their care encounter as possible [. Data tools are translated into the official languages of the study countries and pilot tested for consistent meaning and relevance to the setting. Data collectors are also able to communicate in local languages. The Satisfaction of Employees in Health Care (SEHC) survey is a validated tool to assess staff satisfaction. It was first developed and validated in a low-income country (Ethiopia) [] and later successfully validated in a high-income country (USA) [].

We use a mix of paper and electronic data (ODK [) collection tools. Data collectors are trained to minimise error. Tools are piloted before implementing. ODK data is regularly stored and sent to secure servers, as soon as data collectors reach their office base. Data from paper tools is double entered and compared and sent to secure servers. Each data collection tool has its corresponding electronic database that is cleaned and submitted to the analyses. All data is anonymised at the point of data collection or as soon as possible in the data management process. Data is labelled with an arm code (e.g. ' $\mathrm{A}$ ' or ' $\mathrm{B}$ ') without any further information allowing to disclose which data items belong to the intervention or to the control arms, ensuring blinding during data analyses.

Quality will be assured through several mechanisms: piloting of data collection tools; thorough training of field workers; checking missing data related; double, independent data entry from papers into digital databases; early descriptive analyses to detect potential outliers; fieldworkers tracking and supervision.

\section{Data analysis}

The analysis will be carried out for each country separately, and based on intention-to-treat.

At baseline, data on population and health facility characteristics (i.e. basic demographic characteristics of population and health workers, professional profile of health workers, health facility size and services) will be produced and presented. If large imbalances are detected at baseline, this information can be used to adjust the effect estimate comparisons [].

The analyses vary for the different primary outcomes due to the unit of measurement and levels of clustering, the type of variable, and whether measurements were taken at baseline and endpoint or endpoint only. We use regression models to allow us to estimate the effect of the outcome while flexibly accounting for these issues and allowing adjustment for potential confounders.

Logistic regression will be used for the binary variables: vaccine adherence is measured by determining whether each vaccine due was received, and treatment appropriateness by whether each treatment was correctly prescribed. Data concordance and diagnostic scope are count variables and may be analysed with Poisson regression, depending on their distribution. The regression model for HW satisfaction will depend on how it is distributed.

The outcomes have different levels of clustering (children or consultations, HW, HF). We will account for these levels of clustering by including random effects in the regression models.

Four of the primary outcomes are measured at baseline and end-line. The effect of the intervention will be estimated using an interaction term between arm and survey in the regression models: ie is the change in the outcome between baseline and follow-up in the intervention arm different to the change between baseline and

Page $11 / 16$ 
follow-up in the control arm. The effect of HW satisfaction, measured only at end-line, will be estimated as the difference between the intervention and control arm.

All estimates for the effect of the intervention will be presented with $95 \%$ confidence intervals. The analyses will be carried out using R [.

\section{Measures to minimise bias}

Statistical analyses will be carried out blindly, without knowledge of what health facilities or population in the catchment area belong to the intervention or control groups. Only when the analysis code is considered as definitive and fixed, will results be shared with the wider investigators team and the arms for health facilities and population will be disclosed.

Outcome measurement bias may take place where data from the HIS, which is the focus of the intervention, is used to measure outcomes. However, we will minimise this by assessing population based outcomes at household level.

Contamination (i.e. the intervention affects individuals or units assigned to the control arm) may happen via the exchanges between health workers from health facilities in both arms; for example: in monthly district data quality meetings, managerial meetings; or through inputs from supervisors who influence control health facilities with intervention tips encountered in health facilities of the intervention arms. One mechanism to address this issue is using a district-based cluster randomisation scheme. However, we consider that (i) contamination, despite increasing the awareness of health works in control health facilities, will hardly influence the decision making mechanisms that the HIS intervention focuses on; and (ii) randomisation at the level of district poses additional challenges that are not worth the marginal benefit of reducing a doubtful contamination [.

The spill over effect (i.e. benefits of the intervention extend beyond their direct recipients) [ may take place in higher levels of the health systems; e.g. districts data managers and programme managers may experience the benefits of better structured and more timely data produced in health facilities in the intervention arms. The trial will have no capacity to quantitatively account for spill overs at higher levels of the system, due to the limited number of higher level administrative areas that will be involved in the trial. However, through process indicators, we will consider potential benefits and harms of the intervention at higher levels of the system.

A challenge is the Hawthorne effect (i.e. observer effect). Both health workers in the intervention and in the control sites will have an awareness of being observed as data collection activities will be at the same level of intensity in the two arms. Therefore, there should be no differential effect.

Analyses will be based on the intention-to-treat. It is important to closely monitor if the intervention HFs consistently use the new HIS tools and approaches. The data collection team and the trial monitoring team will check if old forms are still being used in the intervention health facilities. However, we do not expect health facilities to migrate between intervention and control arms, or vice versa, due to feasibility issues. On the other hand, some household members in a given catchment area may decide to seek for health care in a health facility belonging to another trial arm. In these cases, households will be analysed as belonging to the original trial arm.

\section{Discussion}


This is one of the very few studies assessing the effects of health systems interventions using experimental study designs [. Most of the experimental studies on HIS are circumscribed to specific health care areas (e.g. tuberculosis, vaccination, cardiovascular disease) and very few have a system-wide approach (e.g. PHC) [28]. This is the only experimental study we are aware of, focusing on paper-based HIS.

To date, some modifications to the protocol have taken place. In Côte d'Ivoire, we decided to select study areas close to the research institution base on logistics and practical reasons, instead of selecting an area in the north of the country, where poorer health indicators have been described. In Mozambique, the low density of HF per population implied extremely vast distances between HF and this, coupled with the rainy season, made the trial unfeasible in the originally selected Nampula province. After consultations, we decided to move the trial to the province of Inhambane and cancel the household survey. The allocation of HF to the intervention and control arms was completed using random number generation.

Experimental studies for health systems interventions are sometimes dismissed because of their limited capacity to provide reliable explanations of complex health system issues []. While we acknowledge these limitations, there is also a need for more robust evidence on the effects of these types of health system interventions [ and there are also good examples of experimental studies reporting findings that can make it to the policy arena [. When embarking on this research, we considered the type of evidence required to contribute to systematic reviews [], guidance development [] and eventually recommendations for policy and practice []. Furthermore, we have embedded the CRCT in a comprehensive research context which includes systematic reviews of the literature and qualitative research, and we are also looking at explanatory outcomes within the CRCT itself. We believe that this approach will provide a more comprehensive picture of what has happened with the PHISICC tools used by HW and why.

We acknowledge the challenges of carrying out research on health care provided to remote, rural communities (in this case in Sub-Saharan Africa). However, it is only in these remote areas where research about their specific problems and needs can take place. Challenges included long distances, poor conditions of roads, unreliable communications and limited food and accommodation services, all of them to be proactively handled to keep the quality of work and the morale of researchers and collaborators (See picture: additional file 2).

The engagement and ownership of partners within this research has also been instrumental in order to plan and implement the CRCT. The intervention actually targets a governmental sub-system (the HIS) for which we required more than permission but also endorsement and active support. We achieved this level of collaboration by ensuring the participation of key stakeholders in key phases of the whole project, from inception till the implementation of the last phases, through frequent communication and workshops.

We are aware of the current trends towards digitalization of HIS. However, WHO recommendations on the matter are weak since the underlying evidence to support these recommendations is inconsistent [. The principles and methodological approaches in PHISICC can be applied to the development of any technological solution, being on paper, digital or mixed. PHISICC, is not mainly about technologies to support data, but rather about a change in paradigm where life-saving decisions by frontline health workers are at the centre of the intervention; facilitating as well the information requirements of higher levels in the health system.

We expect that the results of the trials, both quantitative and qualitative, will be able to inform policies on how to make HIS responsive to providers' decision-making needs, particularly in health services where the most 
vulnerable live.

Declarations

\section{Abbreviations}

BMGF Bill \& Melinda Gates Foundation

CIV Côte d'Ivoire

CRCT Cluster Randomised Controlled Trial

sd Standard deviation

HF Health Facility

HW Health worker

MOZ Mozambique

NGA Nigeria

WHO World Health Organisation

\section{Declarations}

\section{Ethics approval and consent to participate}

The protocol has been reviewed by the following ethical boards: Comité National Ethique des Sciences de la Vie et de la Santé (CNESVS), reference: 024-19/MSHP/CNESVS-kp (Côte d'Ivoire); Comité Institucional de Bioética para Saúde da Universidade Lúrio, reference: 16.2/Julho/CBISUL/19 (Mozambique); Secretary, Government of Cross River State of Nigeria, Ministry of Health, Calabar Health Research Ethics Committee, reference: CRS/MH/HREC/018/Vol. V1/151 (Nigeria) and Ethikkommission Nordwest- und Zentralschweiz (EKNZ), reference: 2018-01059 (Switzerland).

During the household survey, the informed consent form is presented to the person considered to be the head of the household at the time of the visit. Health workers are also asked to give their consent.

\section{Consent for publication}

Not applicable

\section{Availability of data and materials}

Not applicable 


\section{Competing interests}

The authors declare that they have no competing interests.

\section{Funding}

Bill \& Melinda Gates Foundation - The programme officer has commented on the methods of the study and suggested best ways to adapt it to BMGF grant scope.

\section{Authors' contributions}

$\mathrm{XBC}, \mathrm{AOI}, \mathrm{AM}, \mathrm{RBY}$ and $\mathrm{CAU}$ prepared the proposal for the funding agency, conceived the study and produced the data collection tools. SG ensured the regulatory, ethical and trial monitoring components. AR developed the analytical approaches and made the sample size calculations. RBY, MS and SB adapted the protocol to the context of Côte d'Ivoire and managed the administrative and ethical approvals in the country; AOI, NE, OK, ANN and $A B G$ likewise in Nigeria; AM, SML and GM, in Nampula province (Mozambique); JS and TM adapted the protocol and acquired ethical and administrative clearances for Inhambane province (Mozambique). DB is chair of the PHISICC Technical Advisory Group (TAG) and has coordinated multiple formal and informal inputs. LKK and DB have advised on the adequacy of the study protocol within the overall PHISICC proposal and TAG advices. All country teams participated in PHISICC workshops and ensured that the protocol was suitable to countries realities; developed data collection tools and training materials. They are responsible for the implementation of the trial in each country. XBC drafted the first version of the manuscript. All authors commented on several versions of the manuscript.

\section{Acknowledgements}

Members of the PHISICC Technical Advisory Group, chaired by David Brown: Blanche Anya (WHO AFRO), Abdallah Bchir (former Gavi), Marta Gacic-Dobo (WHO HQ), Richard Greffé (AIGA), Pamela Mitula (WHO AFRO), Sandy Oliver (UCL Institute) and Chris Wolf (BGMF).

Research collaborators: Momade Ali, Celso Belo, Bassirou Bonfoh, Lisa Diallo, John Ferreira, Bernard Guessanbi, Caitlin Jarrett, Inza Koné, Felix Malé, Kouadio M'bra, David O’Donnell, Damaris Rodríguez (Sonder Collective), Melanie Wendland and Meike Zuske (Swiss TPH).

\section{Authors' information (optional)}

\section{References}

\section{Figures}




\section{Remote rural areas}

$\mathrm{N}$ : total number of health facilities in study areas

- Côte d'Ivoire (CIV): Adzopé, Agboville, Sinkensi and Tiassalé

$\mathrm{N}=113$

- Mozambique (MOZ): Funhalouro, Govuro, Homoine, Inhambane, Inharrime, Inhassoro, Mabote, Maxixe and Panda

- Nigeria (NGA): Yala Local Government Authority

$$
N=78
$$

$\mathrm{N}=92$

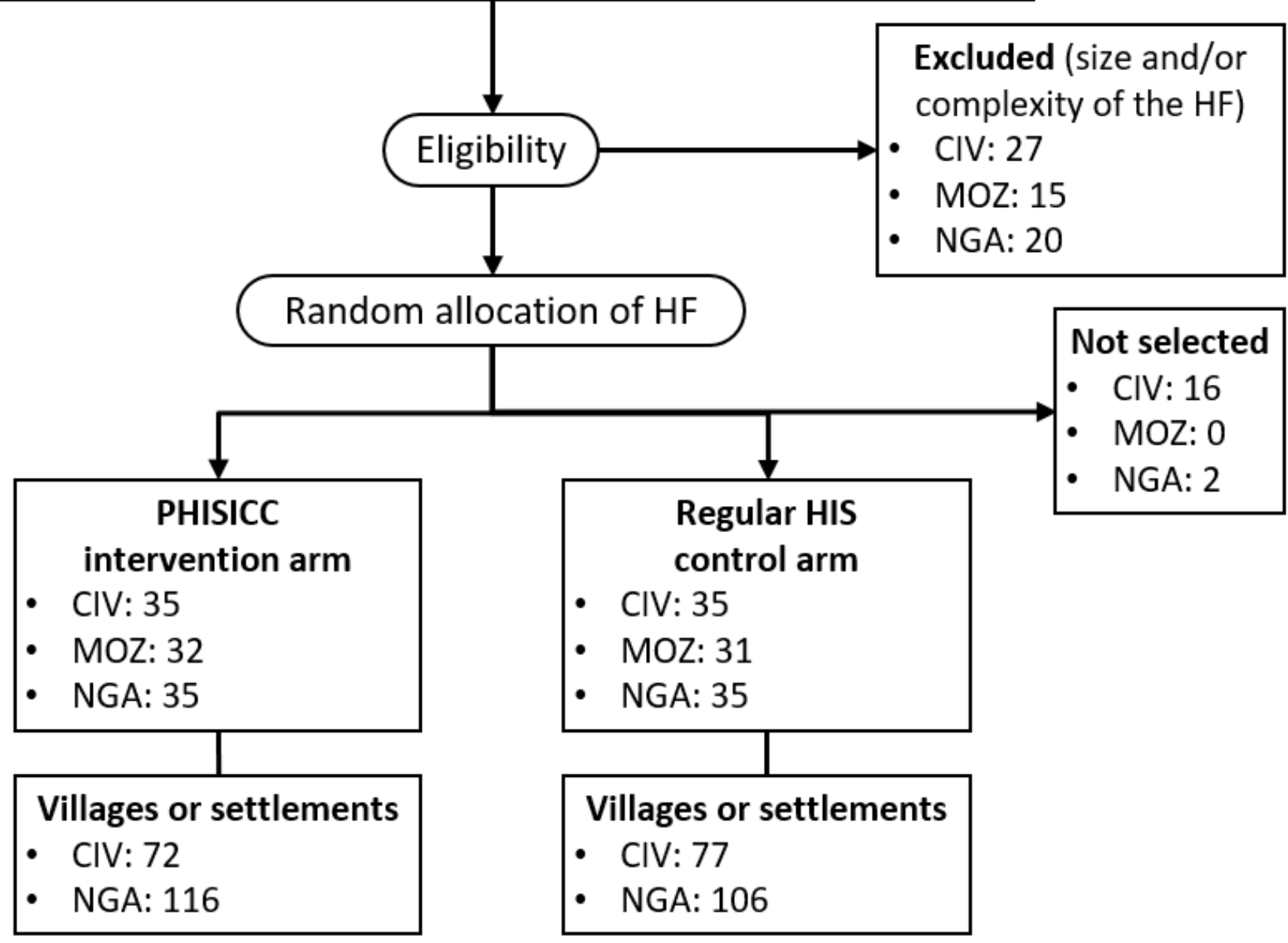

Figure 1

CONSORT diagram: trial flow chart.

\section{Supplementary Files}

This is a list of supplementary files associated with this preprint. Click to download.

- PublicationFilesphisicc50protrctaddfile1consort.docx

- PublicationFilesphisicc50protrctaddfile2.jpg 